\title{
Erratum to: Egg collection from hatchery-reared broodstock of spotted halibut Verasper variegatus treated with LHRH analog
}

\author{
Daisuke Shimizu • Yuichiro Fujinami •
}

Sayumi Sawaguchi · Takahiro Matsubara

Published online: 10 October 2012

(C) The Japanese Society of Fisheries Science 2012

\section{Erratum to: Fish Sci}

DOI 10.1007/s12562-012-0524-2

The article cited above was incorrectly categorized. The correct category is Original Article, not Review Article, with a subject area of Aquaculture.

The online version of the original article can be found under doi:10.1007/s12562-012-0524-2.

D. Shimizu $(\bowtie)$

Tohoku National Fisheries Research Institute, Fisheries Research Agency, Shiogama, Miyagi 985-0001, Japan

e-mail: dshimizu@affrc.go.jp

Y. Fujinami

Miyako Station, Tohoku National Fisheries Research Institute, Fisheries Research Agency, Miyako, Iwate 027-0097, Japan

e-mail: fujinami@affrc.go.jp

S. Sawaguchi

Hokkaido National Fisheries Research Institute, Fisheries

Research Agency, Kushiro, Hokkaido 085-0802, Japan

e-mail: saysaw@fra.affrc.go.jp

T. Matsubara

South Ehime Fisheries Research Center, Ehime University,

Ainan, Ehime 798-4292, Japan

e-mail: matsu@agr.ehime-u.ac.jp 